\title{
Ion-pair reversed-phase high-performance liquid chromatography of algal chlorophylls
}

José L. Garrido ${ }^{\mathrm{a},}$. Manuel Zapata ${ }^{\mathrm{b}}$

${ }^{a}$ Instituto de Investigacions Mariñas, CSIC, Av. Eduardo Cabello 6, 36208 Vigo, Spain

${ }^{b}$ Centro de Investigacions Mariñas. Conselleria de Pesca, Xunta de Galicia, Apdo. 208, 36600 Vilagarcia de Arousa, Spain

*Corresponding author.

\begin{abstract}
The chromatographic behaviour of algal chlorophylls under ion-pair reversed-phase liquid chromatography conditions has been tested either on monomeric and polymeric octadecylsilica columns. Three five-carbon amino compounds (pentylamine, piperidine and pyridine), differing in their molecular shapes and chemical properties, have been used as counterions. The monomeric octadecylsilica column did not allow the separation of chlorophylls $\mathrm{c}_{1}$ and $\mathrm{c}_{2}$ with any amine, but separated chlorophyllide a, chlorophyll $\mathrm{c}_{3}$ and $\mathrm{Mg}$-divinylpheoporphyrin $\mathrm{a}_{5}$ when pyridinium acetate was used as ion pair agent. The polymeric octadecylsilica column separated chlorophylls $\mathrm{c}_{1}$ and $\mathrm{c}_{2}$ with the three counterions, achieving the highest retention and overall separation of acidic chlorophylls when pyridinium was employed as counterion.
\end{abstract}

Keywords: Water analysis; Chlorophylls; Amines; Ion pairing reagents

\section{Introduction}

The determination of photosynthetic pigments in natural waters gives valuable information about the biological activity in different environments [1-3]. The chlorophyll pigments (chls) of marine photosynthetic organisms exhibit a wide range of polarities, from the very non-polar phytol-esterified chls a, b and c-like to the polar chls $\mathrm{c}_{1}, \mathrm{c}_{2}, \mathrm{c}_{3}, \mathrm{Mg}$-divinylpheoporphyrin a5 monomethyl ester (Mg-DVP) and the various chlorophyllides, which contain a free acid function [4]. Many of them are structurally very similar, in certain cases differing from one to another in no more than the position of a double bond (Fig. 1). 
The first high-performance liquid chromatographic (HPLC) separation of acidic chlorophylls employed a polyethylene column eluted with a water-acetone gradient [5]. This method, however, did not allow the simultaneous separation of polar and non-polar chls in a single run.

Saitoh et al. [6] employed an octadecyl-bonded vinyl alcohol polymer (ODP) column and an acidic mobile phase obtaining a partial resolution of chls $\mathrm{c}_{1}$ and $\mathrm{c}_{2}$. To separate acidic and esterified chls the same authors developed a column-switching technique that combines two columns, ODP and octadecylsilica (ODS), with the same mobile phase under isocratic conditions [7]. As chls are acid-labile [8], Shioi et al [9] applied the neutral mobile phases proposed by Garrido and Zapata [10] for the gradient elution separation of acidic and esterified chlorophyll biosynthetic intermediates on an ODP column.

The separation of chls $c_{1}$ and $c_{2}$ on bonded silica columns was first achieved by Kraay et al. [11] using a high-carbon-load $\mathrm{C}_{18}$ stationary phase. The use of polymeric ODS stationary phases, known to exhibit high selectivities towards isomeric compounds with different molecular shapes [12] allowed the complete separation of polar and non-polar chls from chromophyte algae [13]. A similar column was used for the simultaneous separation of most algal polar chls along with the non-polar chls $\mathrm{a}, \mathrm{b}$ and the phytolsubstituted c-like pigment [10], allowing the detection and isolation of several new chls [14]. Polymeric ODS phases were also used (at various column temperatures) for the analysis of chls and carotenoids from marine phytoplankton [15-17].

All the above-cited methods using ODS materials as stationary phases employ ammonium acetate-buffered eluents, based on that first developed by Zapata et al. [2]. However, the selection of different counterions can be used to modulate the retention and separation selectivity of ionic solutes [18]. It could be expected that, with polymeric ODS phases, both variables can be affected by the molecular shapes of different counterions. In this article we study the effect of three organic amines on the retention of acidic chlorophylls either on monomeric and polymeric ODS columns.

\section{Experimental}




\subsection{Conditions}

Methanol, acetonitrile and acetone HPLC grade and pentylamine, piperidine, pyridine and acetic acid (analytical grade) were from Merck (Darmstadt, Germany).

Aliquots of sample solution $(200 \mu 1)$ were injected into a HPLC system based on that described by Garrido and Zapata (1993): a Beckman (Fullerton, CA, USA) System Gold chromatograph including a Model 126 programmable solvent module, a Model 210 injection valve and a Hitachi (Tokyo, Japan) F 1050 spectrofluorimeter (excitation wavelength $440 \mathrm{~nm}$, emission wavelength $660 \mathrm{~nm}$ ) interfaced with a Beckman 406 analog interface module. Columns were thermostatted (at $27^{\circ} \mathrm{C}$ ) with a Beckman Model 235 column heater.

The columns were a Vydac $201 \mathrm{TP}(250 \mathrm{X} 4.6 \mathrm{~mm}$ I.D., polymeric octadecylsilica, $5 \mu \mathrm{m}$ particle size, 300 A pore size) (The Separation Group, Hesperia, CA, USA) or an Ultrasphere $\mathrm{C}_{18}(250 \times 4.6 \mathrm{~mm}$ I.D., monomeric octadecylsilica, $5 \mu \mathrm{m}$ particle size, $80 \mathrm{~A}$ pore size) (Beckman).

Eluents were: (A) methanol-ion-pair solution ( $0.025 \mathrm{M}$ organic amine, $\mathrm{pH}$ adjusted to 5.0 with acetic acid) $(80: 20, \mathrm{v} / \mathrm{v})$ and $(\mathrm{B})$ acetonitrile-acetone $(70: 30, \mathrm{v} / \mathrm{v})$. Three different ion-pair solutions were employed, each containing one of the organic amines pentylamine, piperidine or pyridine. A linear gradient from 0 to $100 \% \mathrm{~B}$ was pumped during $28 \mathrm{~min}$, followed by an isocratic hold at 100\% B during a further $5 \mathrm{~min}$. The initial conditions were recovered in $3 \mathrm{~min}$ by a reversed linear gradient. The flow-rate was $1.2 \mathrm{ml} / \mathrm{min}$.

Pigment standards were obtained from unialgal cultures of three species of known pigment composition: the diatom Skeletonema costatum, the prymnesiophyte Prymnesium parvum and the prasinophyte Micromonas pusilla. Peak identity was confirmed as previously described [10].

Natural seawater samples (1.51) were filtered and extracted as described in Ref. [10]. 


\section{Results and discussion}

The three amino compounds used as counterions in this study show some properties making them suitable for the chromatographic analysis of acidic chlorophylls: they are liquids with adequate viscosities, miscible with water and most organic solvents used in liquid chromatography (higher amines show limited solubilities in water), they are relatively volatile so they can be easily removed after preparative separations [19] and, even being highly absorbing in the ultraviolet, they do not interfere the detection of the pigments (visible light absorption or fluorescence). They are inexpensive and readily available.

For our purposes, their differences in molecular shape were specially interesting. Pyridine is strictly planar and rigid, the saturated cyclic piperidine molecule represents an intermediate stage and pentylamine is the most flexible and voluminous of the three structures.

The strengths as bases of pentylamine, piperidine and pyridine are very different [19]. The weakest base is pyridine, with a $\mathrm{pK}_{\mathrm{a}}$ of the conjugate acid, pyridinium ion, of 5.19. In consequence the $\mathrm{pH}$ of the eluents was adjusted to a value of 5.00. No alteration of any chlorophyll was observed throughout this work.

The separation of polar chls, especially of the mono- and divinyl analogues chl $\mathrm{c}_{1}$ and $\mathrm{chl} \mathrm{c}_{2}$ seems to depend on the nature of the stationary phases (Fig. 2 and Fig. 3). As it has been previously observed for ammonium acetate buffered mobile phases [10], no separation of these two chls could be achieved with the monomeric ODS column (Fig. 2 ), in spite of the higher retentions attained with the organic amines. When the mobile phase contained pentylammonium or piperidinium ions, the coelutions of chlorophyllide a with chl $\mathrm{c}_{3}$ and of Mg-DVP with chls $\mathrm{c}_{1}$ and $\mathrm{c}_{2}$ were observed. However, the pyridinecontaining mobile phase showed an enhanced selectivity, allowing the separation of chlorophyllide a, chl $\mathrm{c}_{3}$ and Mg-DVP from the critical pair of chls $\mathrm{c}_{1}$ and $\mathrm{c}_{2}$. This selectivity change can be explained considering the groups in which chromatographic solvents have been classified [18]. In addition, the aromatic character of pyridine allows p-interactions with the highly aromatic chlorophyll macrocycle. This ability of pyridinecontaining eluents to separate certain acidic chls on monomeric phases could be 
exploited in the pigment analysis of species with no simultaneous occurrence of chlorophylls $\mathrm{c}_{1}$ and $\mathrm{c}_{2}$ (prasinophytes, some cryptophytes, peridinin-containing dinoflagellates, certain prymnesiophytes, etc...) [5]. The behaviour of the esterified chlorophylls is not affected by the presence of a different amine in the mobile phase, the phytol substituted chl c from Prymnesium parvum eluting always between chls $\mathrm{b}$ and a.

The effect of the three different ion-pairing agents is greater when polymeric octadecylsilica is used as stationary phase (Fig. 3). The general elution pattern for chlorophyll pigments on polymeric octadecylsilica columns has been explained in terms of their overall molecular polarity and of their molecular shape [10]. Accordingly, the voluminous chlorophyllide a appears as the first eluting peak in the chromatograms, well resolved from other pigments. At the other end, the phytylated chlorophylls kept their retention times almost constant regardless of the mobile phase employed, with the planar phytol-substituted chl c .eluting after chlorophyll a. Chls $\mathrm{c}_{1}$ and $\mathrm{c}_{2}$ are well separated with this stationary phase with the three counterions, whereas Mg-DVP and chl $c_{3}$ coelute under any condition assayed. The retention of acidic chlorophylls increases as the counterion is more planar, reaching its highest value when the mobile phase contains the completely planar pyridinium ion. This increase in retention is paralleled by an enhanced separation of chl $\mathrm{c}_{1}$ from $\mathrm{chl}_{2}$ (Fig. 3). This behaviour agrees both with the "slot model", that explains the shape selectivity shown by polymeric octadecylsilica phases [20,21] and with the paired-ion or dynamic exchanger models, proposed to describe the retention in ion-pair chromatography [22].

When a shorter gradient program is applied to the system with the polymeric column and the pyridinecontaining eluent, the analysis of a pigment extract from a natural seawater sample (a sample that would take more than $40 \mathrm{~min}$ to be analyzed by the column-switching method [71]) is completed in less than 20 min (Fig. 4). Under these conditions, standard solutions of the different pigments were injected and the corresponding calibration graphs were prepared by plotting concentrations against peak areas. The responses were linear in the range $0.05-1.50 \mathrm{pug} / \mathrm{ml}$ for all the chlorophylls assayed. The detection limits (calculated on the basis of $3 \sigma$ ) were $4.90 \mu \mathrm{g} / \mathrm{ml}$ for chl a, $2.62 \mathrm{ng} / \mathrm{ml}$ for chl b, $1.43 \mathrm{ng} / \mathrm{ml}$ for chl $\mathrm{c}_{1}$ and $1.31 \mathrm{ng} / \mathrm{ml}$ for $\mathrm{chl}_{2}$. 
The chromatogram in Fig. 4 corresponds to a natural sea-water sample from Ria de Arousa (Galician coast, NW Spain). The relative amounts of chls $c_{1}(0.197 \mu \mathrm{g} / 1 \mathrm{sea}$ water $)$ and chl $c_{2}(0.819, \mu$ gll sea water $)$, as well as the low levels of chl b $(0.128 \mu \mathrm{g} / \mathrm{l}$ sea water $)$ and chl $c_{3}\left(0.128 \mu \mathrm{g} / \mathrm{l}\right.$ sea water. estimated as chl $\left.\mathrm{c}_{2}\right)$ and the high concentration of chl a $(2.320 \mu \mathrm{g} / 1$ seawater $)$ are typical of a phytoplanktonic community dominated by diatoms and dinoflagellates.

References

[1] R.F.C. Mantoura and C.A. Llewellyn, Anal. Chim. Acta, I51 (1983) 297.

[2] M. Zapata. A.M. Ayala. J.M. Franco and J.L. Ganido, Chromatographia. 23 (1987) 26.

[3] S.W. Wright. S.W. Jeffrey, R.F.C. Mantoura, C.A. LLewellyn. T. Bjomland, D. Repeta and N. Welschmeyer, Mar. Ecol. Prog. Ser. 77 (1991) 183.

[4] D.F. Millie, H.W. Paerl and J.P. Hurley, Can. J. Fish. Aquat. Sci., 50 (1993) 2513.

[5] S.W. Jeffrey, in J.C. Green, B.S.C. Leadbeater and W.L. Diver (Editors). The Chromophyte Algae: Problems and Perspectives. Clarendon Press, Oxford, 1989, Ch. 2, p. 13.

[6] K. Saitoh, 1. Awaka and N. Suzuki. J. Chromatogr. A, 653 (1993) 247.

[7] K. Saitoh, I. Awaka and N. Suzuki, J. Chromatogr. A, 693 (1995) 176.

[8] P.H. Hynninen. in H. Scheer (Editor). Chlorophylls, CRC Press, Boca Raton, FL, 1991. Ch. 1.7, p. 145.

[9] Y. Shioi, K. Watanabe. K. Takamiya, J.L. Garrido and M. Zapata, Anal. Biochem.. $231(1995) 225$.

[10] J.L. Garrido and M. Zapata. J. High Resolut. Chromatogr., 16 (1993) 229.

[11] G.W. Kraay. M. Zapata and M.J.W. Veldhuis, J. Phycol., 28 (1992) 708

[12] L.C. Sander and S.A. Wise, J. Chromatogr. A. 656 (1993) 335.

[13] J.L. Garrido and M. Zapata, Chromatographia. 35 (1993) 543.

[14] J.L. Garrido, M. Zapata and S. Muiiiz, J. Phycol., 31 (1995) 761.

[15] L. Van Heukelem, A.J. Lewitus, T.M. Kana and N.E. Craft, J. Phycol. 28 (1992) 867

[16] L. Van Heukelem, A.J. Lewitus, T.M. Kana and N.E. Craft, Mar. Ecol. Prog. Ser. 114 (1994) 303 
[17] K. Van Lenning, J.L. Garrido. J. Aristegui and M. Zapata, Chromatographia, 41 (1995) 539.

[18] L.R. Snyder and J.J. Kirkland, Introduction to Modem Liquid Chromatography, Wiley, New York, 2nd ed., 1979.

[19] S. Budavari (Editor), The Merck Index, Centennial Edition, Merck, Rahway, 1989.

[20] S.A. Wise and L.C. Sander, J. High Reaolut. Chromatogr. Chromatogr. Commun.. 8 (1985) 248.

[21] L.C. Sander and S.A. Wise, LC.GC, 8 (1990) 378.

[22] L.R. Snyder, J.L. Glajch and J.J. Kirkland, Practical HPLC Method Development. Wiley, New York, 1988. 
Fig. 1. Structures of chlorophylls from marine microalgae.

Fig. 2. Chromatograms of chlorophyll standards on the monomeric ODS column (Ultrasphere $\mathrm{C}_{18}$ ) using mobile phases containing $(\mathrm{A})$ pentylamine, $(\mathrm{B})$ piperidine and (C) pyridine. Peak identification: $1=$ chlorophyllide a; $2=$ chlorophyll $\mathrm{c}_{3} ; 3=\mathrm{Mg}$-DVP; $4=$ chlorophyll $\mathrm{c}_{1} ; 5=$ chlorophyll $\mathrm{c}_{2} ; 6=$ chlorophyll $\mathrm{b} ; 7=$ chlorophyll a allomer; $8=$ chlorophyll a; $9=$ chlorophyll a epimer; $10=$ phytol-substituted chlorophyll c. Retention times are indicated in brackets. For other chromatographic conditions, see Section 2.

Fig. 3. Chromatograms of chlorophyll standards on the polymeric ODS column (Vydac $201 \mathrm{TP}$ ) using mobile phases containing (A) pentylamine. (B) piperidine and (C) pyridine. Peak identification as in Fig. 2. Retention times are indicated in brackets. For other chromatographic conditions, see Section 2.

Fig 4. Chromatogram of a pigment extract from a natural seawater sample on the polymeric column (Vydac $201 \mathrm{TP}$ ). Mobile phases as in Fig. K. Gradient program: 0 to $100 \% \mathrm{~B}$ in $15 \mathrm{~min}$; $\min 15$ to $\min 20,100 \% \mathrm{~B}$. Flow-rate: $1.2 \mathrm{ml} / \mathrm{min}$. Retention times of the different chlorophylls are indicated in brackets. 